\title{
Reasons for Missed Visits and Influence of Phone Call Reminders on Retention of HIV-Infected and Uninfected Children Followed in Cameroon: The ANRS-PEDIACAM Cohort
}

Francis Jaudel YUYA SEPTOH ( $\sim$ francisyuya@gmail.com )

CENTRE PASTEUR DU CAMEROUN https://orcid.org/0000-0002-5513-4490

Jules Brice Tchatchueng

Centre Pasteur Cameroon

Francis Ateba Ndongo

Mother and Child Center of the Chantal Biya Foundation

Ida Calixte Penda

Laquintinie Hospital

Georgette Guemkam

Mother and Child Center of the Chantal Biya Foundation

Suzie Tetang

Essos Hospital Center

Derboise Gweha

Essos Hospital Center

Angèle Bense

Mother and Child Center of the Chantal Biya Foundation

Astrid Moukoko

Laquintinie Hospital

Casimir Ledoux Sofeu

Centre Pasteur Cameroon

Albert Faye

France Université Paris Diderot

Josiane Warszawski

CESP UMR Villejuif

Mathurin Cyrille Tejiokem

Centre Pasteur Cameroon

\section{Research}

Keywords: HIV-uninfected children exposed or not, non-compliant, phone calls, reasons for missing visits 
DOI: https://doi.org/10.21203/rs.3.rs-99602/v1

License: (c) (1) This work is licensed under a Creative Commons Attribution 4.0 International License. Read Full License 


\section{Abstract}

\section{Background}

As many longitudinal studies, follow-up in the ANRS-PEDIACAM study is disrupted by repeated absences of participants to scheduled visits. This lead to missing data which influence the quality of results. We describe reasons for participants absence or non-compliance (NC) and assess the influence of phone call reminders (CR) on retention in care.

\section{Methods}

From November 2007 to 2011, 611 infants divided in three groups were included and followed in three referral hospital in Cameroon: HIV-infected children followed from the first week of life or not but diagnosed before seven month of life ( $n=210)$, HIV-uninfected children born to HIV-infected (HEU) $(n=205)$ mothers or not (HUU) $(n=196)$. From 2014 to 2017, CR were reinforced to record reasons of missing visits. we used frequency, chi-square or Fisher test for categorical variables; means, median (IQR) and non-parametric Kruskal Wallis test for continuous variables. A multistate transition modelling approach was used to analyse the retention care cascade. The R software was used to perform all statistical analysis.

\section{Results}

During the study period, $45.1 \%$ (246/546) of children were NC at least once of which $16.3 \%(25 / 153), 58 \%$ (116/200), 54.4\% (105/193) respectively among HIV-infected, HEU and HUU-children. Among NC, 69.5\% (171/246) has been reachable at least once and $22.2 \%$ (38/171) of them returned to follow-up after a median delay of 32 days (IQR: [2.0 - 110]); 44.4\% (109/246) were not seen throughout the study period (HIV-infected: 12/153, HEU-children: 57/200, HUU-children: 40/193). A total of 276 reasons have been reported among $54 \%(115 / 213)$ of the NC, mainly related to delocalisation $(30.4 \%)$, lack of time $(23.6 \%)$, wish to stop follow-up (11.6\%), travelling (9.8\%), schooling (9.4\%), forgetting (7.2\%). Comparing before and after period, CR allowed to divided by three adjusted relative risk ratio to miss one clinical visit (RRR [CI]: 0.35[0.24 - 0.52]), However CR effect was not significant among children who are non-compliant.

\section{Conclusion}

Our finding suggest that maternal HIV and socio-economic status are related to attendance of children in HIV care. Also, the CR are an effective strategy to improve attendance. However, to make this strategy effective for children who are non-compliant, it needs to be strengthened by community monitoring.

\section{Introduction}

The Human Immunodeficiency Virus (HIV) infection remains a major problem in sub-Saharan Africa countries with bear the heaviest global burden with nearly 67\% of persons living with HIV (PLWH) in 2019 (1). The scale-up of HIV testing and antiretroviral therapy (ART) has helped to control the disease and 
improve the survival and quality of life of PLWH (2). These global efforts had also an impact on the prevention of new HIV infection among children born to HIV infected mother, leading to a $60 \%$ reduction of the number of HIV pediatric infection in sub-Saharan Africa between 2002 and 2013 (3) and increasing of the number of HIV-exposed uninfected (HEU) children.

Coverage of ART among HIV-infected children has also improved during these last years. In $2017,52 \%$ of HIV-infected children received ART. However, ART programmatic success requires both a sustainable supply of ART at the facility level and lifelong adherence to treatment by PLWH (4). Poor ART retention in care and adherence may lead to virological failure, reducing future therapeutic options $(5,6)$, in the context of very limited access to the second and third lines regimens, and a hindrance in achieving UNAIDS 90-90-90 targets set for $2020(7,8)$. Retention in care also concerned HEU-children as it has been demonstrated that they are at high risk of mortality and morbidity compared to children born by HIVuninfected mother (HUU) during the first year of life (9-11). The mechanisms of occurrence are not fully understood (12-14).

The ANRS-PEDIACAM cohort is constituted of HIV-infected, HEU and HUU children groups followed in Cameroon. As most prospective studies, it is threatened in its management by episodic and repeated absences to scheduled visits $(8,15)$. In that cohort, the proportion of "Non-compliant (NC)" children increased gradually during follow-up, especially among HIV-uninfected children. This could have consequences not only on their health, but also on the analysis and interpretation of the results. The reminders methods based on mobile telephony tools might help in improving patient adherence in health facilities (16-18). Based on that hypothesis, we reorganized and intensified the use of phone calls within the ANRS-PEDIACAM study from April 2014.

The aims of this study were to describe the reasons for missing scheduled visits and to assess the influence of phone call reminders (CR) on retention.

\section{Methods}

Study setting: the ANRS-PEDIACAM cohort study

This study was developed within the framework of the ANRS-Pediacam cohort which is an ongoing longitudinal study coordinated by the Centre Pasteur of Cameroon, designed to assess the feasibility of early HIV-diagnosis and early treatment of HIV-infected children in resources limited settings $(19,20)$. From November 2007 to October 2011, a total of 611 children were included in three groups and followed in three referral hospital in Cameroon (the Mother and Child Center of the Chantal Biya Foundation (MCC$\mathrm{CBF}$ ) and the Essos Hospital Center (EHC) in Yaounde, and the Laquintinie Hospital in Douala (LHD)). These groups consist of: HIV-infected ( $n=210)$, HEU-children $(n=205)$ and HUU-children $(n=196)$. Followup was scheduled three-monthly until the age of two years, then six-monthly for HIV-infected children; while HIV-uninfected children were followed six-monthly.

\section{Study population}


Our study population consisted of children followed in the ANRS-PEDIACAM cohort. By April 2014, 65 (10.6\%) died (figure 3) leading to 546 children including 153 HIV-infected, 200 HEU, and 193 HUU considered in this study. Of these children, 160 (29.3\%) were considered as non-compliant.

\section{Intervention phone reminders strategy and data collection}

From April 2014 to April 2017, we issued every week a list of expected children for their visit. Phone calls were conducted by a Clinical Research Assistant as follow:

- At week $i$, all the NC children of the week.

- If not seen, wait two weeks before calling back

- If still not seen restart the procedure immediately for HIV-infected children or wait for two months before restarting for uninfected children.

The topics discussed on phone were: children vital status, reminder of the missed visit, reasons for missing, and taking an appointment to return to care. The collected data were then entered into an Access database.

\section{Data used}

We used socio-demographic data collected at the study inclusion, the subject history of missing visit, the reasons for non-return to the visit for NC children and phone reminder from April 2014 to April 2017.

\section{Outcome definition}

A child was considered to have missed a visit ' $V_{i}^{\prime}$ if he did not come to the expected date of the visit ' $V_{i}$ ', and never returned since that date until his next scheduled visit ' $\mathrm{V}_{\mathrm{i}+1}$ '.

The outcome was "Non-compliant (NC)" defined as children alive or unknown status who missed at least two consecutive visits.

\section{Statistical analysis}

Socio-demographic parameters and reasons for missed visits were described and compared between groups using respectively frequency, chi-square or Fisher test for categorical variables; means, median (IQR) and non-parametric Kruskal Wallis test for continuous variables. A multistate transition modelling approach was used to analyse the retention care cascade. Follow-up time was discretized into intervals centred on the scheduled visit date for each child. At each time interval, four discrete states were defined using children-level data: seen, alive and not seen only at the current visit (missed visit), alive and not seen since the last scheduled visit (Non-Compliant), and deceased. Figure 1 is a graphical representation of the states and possible transitions. States transition matrix was calculated under the first-order Markov dependence assumption. To evaluate the influence of the call reminder enhancement performed on retention, we use a multinomial regression model (characterized by: retention to the care; duration of 
consecutive missing scheduled visit and mortality) to estimated the Relative Rate Ratio (RRR) (21) associated with each. A p-value of 0.05 or less was considered to be statistically significant. The $R$ software version 3.3.3 was used to perform all statistical analysis.

\section{Results}

\section{Population characteristics}

Of the 546 children considered at the start of this sub-study, 193 (35.3\%) were HUU, 200 (36.6\%) HEU and $153(28.0 \%)$ HIV-infected. Among them, 48.5\% ( $n=265)$ were included at the MCC-CBF, 27.8\% $(n=152)$ at EHC in Yaounde, and 23.6\% $(n=129)$ at the LHD in Douala. The children characteristics at inclusion differed significantly among groups (Table 1). Compared to the HIV-infected group, mothers of uninfected groups (HUU and HEU) were more likely to be educated, employed, and involved in a stable relationship.

\section{Non-compliance to visit}

At the beginning of this study, 29.3\% (160/546) were NC (April 2014), mostly HIV-uninfected children (36.9\% (145/393) vs $9.8 \%$ (15/153), p < 0.001) (Fig. 3). Non-compliance status was also associated with the site of inclusion and follow-up group (Table 1). Among children who were $\mathrm{NC}$ at the beginning of our intervention, 68.1\% (109/160) did not return for follow-up during the entire evaluation period with a median delay from the last visit of 71 months (IQR: [60-83]); respectively, 80\% (12/15), 72.1\% (57/79), $60.6 \%$ (40/66) from the HIV-infected, HEU, and HUU groups. From the 386 compliant children at the beginning, $77.7 \%(300 / 386)$ remained compliant during the study period and the remaining $22.3 \%$ (86/386) became NC with a median cumulative delay from the last visit of 34.2 months (IQR: [27.644.3]). This leads to a total of $35.7 \%(195 / 546)$ NC to visit at the end of the evaluation in April 2017; showing about $6.4 \%$ increased of the number of NC.

A total of $45.1 \%(246 / 546)$ of children had been considered NC at least once including $16.3 \%(25 / 153)$, $58 \%$ (116/200), and 54.4\% (105/193) respectively in HIV-infected, HEU, and HUU groups (Fig. 3).

Figure 2 shows the number of children with available data as a function of the potential length of followup time. The number of children not seen was more important in the HEU and HUU group and increased during follow-up. However, in the HIV-infected group, the gap observed is attributable to mortality in the early follow-up period.

Tables 3 and 4 provide a summary of state transition rates before and after call reminder enhancement (CRE), using all data aggregated over time. Entries in the upper left indicate the cyclic nature of retention in care: among those seen at their current scheduled visit, the rate of retention in care is $84 \%$ and $89 \%$ respectively before and after CRE. Among those not seen only at the last visit, about $47 \%$ and $54 \%$ respectively before and after $\mathrm{CRE}$, become $\mathrm{NC}$ at the current visit. The estimated probability transition matrix shows that children who are NC have a higher risk to not return for follow-up.

\section{Phone calls results}


About 1069 phone calls were made for the 868 visits scheduled to NC children when they were NC during April 2014 to April 2017, among these calls 693(64.8\%) were unreachable. Of the 376(35.2\%) reachable phone calls which resulted in an interview, 168(44.7\%) ended with an appointment and $26.2 \%(44 / 168)$ were subsequently realized (Fig. 4). The proportion of unreachable phone calls increased significantly with a long delay since the last visit (35\% for $\leq 7$ months; $45 \%$ for $7-12$ months, and $65 \%$ for $>12$ months, $p<0.001)$. About $80.3 \%(171 / 213)$ of called children were at least once reachable while $19.7 \%$ $(42 / 213)$ were always unreachable during the evaluation of which $71.4 \%(30 / 42)$ were already $\mathrm{NC}$ before the evaluation period.

\section{Reasons why children and parent's pairs weren't returning for follow-up}

Of the 376 reachable phone calls realized, about $54 \%(115 / 213)$ of contacts provided reasons $(n=276)$ why they did not come back to visits (Fig. 4 and Table 2). These reasons were mainly: changing of residence $(30.4 \%)$, lack of time $(23.6 \%)$, wish to stop follow-up (11.6\%), parent and/or child trip (9.8\%), school attendance by children (9.4\%), forgetfulness (7.2\%), illness or death of a relative $(4.7 \%)$ and $3.3 \%$ other reasons including lack of motivation. This distribution did not change with those of children who were always NC. No significant difference was found between the reasons given in each follow-up group. Two-third of parents whose reason of non-returning for follow-up was changing of residence have always been non-compliant (Table 2).

\section{Impact of phone reminders on retention to care}

Of the 160 NC children at the beginning of this evaluation, 31.8\% $(n=51)$ returned to follow-up (Fig. 3$)$. Among children, whose parents were called and reached at least once, 21.2\% (38/171) returned to followup (Fig. 4). We also noted that among the 2046 visits completed, $32.2 \%(659 / 2046)$ were after the scheduled date of appointment; and among them, 91\% (600/659) were achieved through phone calls.

\section{Factors associated with state transition probability}

Tables 5,6 , and 7 show the effect of covariates on the state transition probabilities in terms of relative rate ratio. Among the key findings:

- The adjusted relative rate ratio (RRR) of death among HIV-infected children who missed their last visit was significantly higher (RRR [CI]: 12.32 [3.54-42.85]) compared to HEU children who were missed at their last visit. The RRR of death among HIV-infected children who were NC at their last visit was significantly higher (RRR [Cl]: 8.76[1.60-47.9]) compared to HEU-children;

- The RRR of death among children who are missed their last visit, whose mothers are housewives or unemployed was significantly higher (RRR [Cl]: 3.24 [1.8-13.0]) compared to children whose mothers have a paid activity;

- The RRR of missing visit among children seen at their last visit was divided by three (RRR [CI]: 0.35 [0.24-0.52]) after enhancement of call reminder. However, call reminder had no significant effect among children who were missed or non-compliant at their last visit; 
- The RRR of missing visit among HIV-infected children who were seen at their last visit was reduced by one third (RRR [Cl]: 0.65 [0.53-0.82]) compared to HEU children who are missed their last visit;

- HIV-infected children (0.14 [0.08-0.23]) and HUU children (0.65 [0.44-0.94]) were less likely to be non-compliant compared to HEU children;

- Children included in the Pediacam cohort in 2011 and seen at their last visit had a higher relative rate ratio (RRR [Cl]: 1.70 [1.11-2.61]) to miss their current visit compared to children included in 2008. The RRR of death among children included in 2009 and who missed their last visit was significantly lower (RRR [Cl]: 0.38 [0.15-0.93]) compared to children included in 2008 who are missed their last visit. Children included in 2009 were less likely (RRR [Cl]: 0.60 [0.33-0.95]) to be non-compliant compared to those included in 2008 (Table 6);

- Children included in LHD were less likely to be non-compliant (RRR [CI]: 0.55 [0.37-0.82]) compared to children included in MCC-CBF. While children included in EHC were most likely to be non-compliant (RRR [Cl]: 1.74 [1.66-2.60]) compared to those included in MCC-CBF;

- The RRR in Table 7 indicates that children from a low-income family $(<50000)$ are most likely to remain non-compliant compared to children from high income family (>150000).

\section{Discussion}

This paper describes the reasons for missing scheduled visits and the influence of phone reminders on missing visit in the ANRS-PEDIACAM cohort from April 2014 to April 2017; which is an ongoing longitudinal study set up for more than ten years. Also, the influence of phone reminders on missing visit state, from inclusion to April 2017 was modelled using discrete time multistate framework characterized by: retention to the care; duration of consecutive missing scheduled visit and mortality.

Overall, about $45 \%$ of children followed had been considered NC at least once during the evaluation period. The proportion of NC was significantly higher (56\%) among HIV-uninfected children particularly among HEU-children (58\%). This observation reinforces those reported by Sidze et al (22) in a previous study conducted in the same cohort which showed that the proportion of lost to follow up children was significantly lower in HIV-infected and HEU-children compare to HUU-children, and only $35 \%$ of the latter could be joined by phone calls. The high proportion of NC among HIV-uninfected children might be related to parent decision. As a longitudinal study, PEDIACAM has consisted of regular clinical visits. The parents whose child is not sick, or shows no signs of illness, may not perceive the importance of regular clinical visits and will even try to stop follow-up as was observed in $90.6 \%(29 / 32)$ of those who show interest to stop. On the other hand, a high proportion of mothers (42\%) of HEU-children were unaware of their HIV status before prenatal HIV screening. The Woman who had not disclosed her own HIV infection status to the family (especially her partner) had difficulties justifying frequent visits with the child to the health facility. Fear of violence and stigma from their families prevented them from utilizing HIV care services (23). 
In this study, phone calls reminder had considered as a search tool on returning to care. The influence of this intervention in improving patient adherence in health facilities had been shown in a similar context $(16,17,24)$. Only $35.2 \%$ of the total phone calls made resulted in a phone interview. The reasons for NC to missed visits were mainly related to geographic mobility of population (relocation, travels), lack of time, and forgetfulness. These reasons are similar to those presented in other study (25). However, in our study, others important reasons like stigma, lack of money, and the cost of transport were not mentioned as indicated in some studies conducted in the resource's limited countries $(22,25-28)$. In fact, in PEDIACAM study, to avoid missing scheduled visits, free medical support and reimbursement of transport fares were brought to the children. This is curious when we find that it's the uninfected children who miss the most visits, and that is why we can think that this stigma reason could have been masked among the answer we collected.

The predominance of relocation (30.4\%) among reasons given in our study could be explained by people dynamic related to study duration. Some of the parents were transferred for professional purpose, others relocated because of economic reasons or modification of the composition of the family with the death of one of the parents. Most of the parents who change the place of residence had children always NC indicating that specific measures should be implemented to tackle such a problem.

Phone call reminders (CR) allowed to reduce significantly adjusted relative risk to miss one scheduled visit to two third. This result shows that $\mathrm{CR}$ are an effective intervention to improve the retention in care. Our results are in line with those recently published in a systematic review of current clinical evidence of CR on retention to care among HIV patients (29). The later used the results from nine studies carried out in four sub-Saharan African countries (Cameroon, Kenya, South Africa, Eswatini), Switzerland, United Kingdom and United States of America. However, this intervention shows weaknesses for children noncompliant. Indeed, a large number of calls performed among non-compliant children remained unreachable throughout the study period.

Multivariate analysis identified others economically and individual's factors associated with a missing visit or non-compliant: HIV infection, low family incomes, mother's occupation. In addition, low-income family live in a precarious situation, which leads to frequent relocation to find a better situation or for seasonal work. This may result in an increase in the distance from the site of care, an increase in the cost of transport and therefore pre-financing difficulties. On the other hand, most of the mothers ( $40 \%)$ of HEUchildren were housewives or unemployed at the inclusion of their child in the study. However, it can be noted that mothers who were housewives or unemployed were not financially independent and therefore needed financial support from a partner or their families to come frequently to clinical visits.

This study has some limitations: the phone calls were the only research tool used in this study for reminders and collection of informations about the reasons for missing visits. More than half of calls performed were unreachable. As a result, reasons for missing visits were not always available, and could only be collected from those who were reachable. The time intervals between clinical visits were irregular throughout the follow-up. In multivariate analysis, we only considered covariables measured at inclusion 
in our study. Indeed, the model was the concern with missing visits, and at these visits, the dynamic data were missing. In this context, the modeling requires a step of the imputation of the missing data beforehand. In a recent paper, Lee and al (21) used a most recent observed value for imputing missing data, however this strategy to deal with missing data is not always appropriate. In future work, comparing this method with other imputation strategies may determine the best way to consider dynamic data in this framework.

\section{Conclusion}

Our findings suggest that maternal HIV infection and socioeconomic status could influence the attendance of children in HIV care. Strengthening counselling of mothers of HIV-uninfected children to better explain the objectives of the study and the reasons of their participation can help to improve the attendance of HEU-children as well as HUU-children. On the other hand, the phone call reminder is an effective intervention to improve attendance to care. However, this intervention is limited among noncompliant children, due to the high proportion of unreachable calls, what indicates that this strategy cannot be used alone and should always be associated to be more effective like community monitoring.

\section{Declarations}

\section{Ethics approval and consent to participate}

The ANRS-Pediacam study was granted ethical approval in Cameroon by the National Ethics Committee and in France by the Biomedical Research Committee of the Pasteur Institute of Paris. The Cameroon Ministry of Public Health gave administrative authorization to start the study. We obtained free and informed consent from all study participants.

\section{Consent for publication}

We give our consent to the publication of this work.

\section{Availability of data and material}

The data and tools of this study remain available and are maintained by our teams.

\section{Competing interests}

We declare that this study is not subject to any conflict of interest.

\section{Funding}

This study was funded by the French National Agency for Research on AIDS and Viral Hepatitis.

\section{Authors' contributions}


Conceived and designed the experiments: TMC AF JW PIC. Analyzed the data: YSF TJB SCL TMC. Wrote the paper: YSF TJB TMC. Coordination of the study: TMC AF PIC JW. Recruitment and infants follow up: PIC GG ANF TS GD BA MA.

\section{Acknowledgements}

We acknowledge the French National Agency for Research on AIDS and Viral Hepatitis for funding this study. We are also grateful to parents who accepted their children to be included in this study. We thank the entire study team at each site for their commitment and excellent collaboration.

\section{References}

1. UNAIDS. Global HIV \& AIDS statistics - 2020 fact sheet. 2020.

2. Trickey A, May MT, Vehreschild J-J, Obel N, Gill MJ, Crane HM, et al. Survival of HIV-positive patients starting antiretroviral therapy between 1996 and 2013: a collaborative analysis of cohort studies. The Lancet HIV. 2017;4(8):e349-e56.

3. Prendergast AJ, Essajee S, Penazzato M. HIV and the millennium development goals. Archives of disease in childhood. 2015;100(Suppl 1):S48-S52.

4. AIDS. UNAIDS 2018 estimates and UNICEF Global Databases of nationally representative populationbased surveys 2010-2017. 2018.

5. Kabogo J, Gupta S, Maina A, Ochwoto M, Omange R, Musoke R, et al. RISK FACTORS OF VIROLOGIC FAILURE AND SLOW RESPONSE TO ART AMONG HIV-INFECTED CHILDREN AND ADOLESCENTS IN NAIROBI. East African Medical Journal. 2017;91(4).

6. Hawkins C, Ulenga N, Liu E, Aboud S, Mugusi F, Chalamilla G, et al. HIV virological failure and drug resistance in a cohort of Tanzanian HIV-infected adults. Journal of Antimicrobial Chemotherapy. 2016;71(7):1966-74.

7. HIV/AIDS JUNPo. 90-90-90: An ambitious treatment target to help end the AIDS epidemic. Geneva, Switzerland: UNAIDS; 2014. 2017.

8. Kalembo FW, Zgambo M. Loss to followup: a major challenge to successful implementation of prevention of mother-to-child transmission of HIV-1 programs in sub-Saharan Africa. Isrn Aids. 2012;2012.

9. Arikawa S, Rollins N, Newell ML, Becquet R. Mortality risk and associated factors in HIV-exposed, uninfected children. Tropical Medicine \& International Health. 2016;21(6):720-34.

10. Slogrove A, Reikie B, Naidoo S, De Beer C, Ho K, Cotton M, et al. HIV-exposed uninfected infants are at increased risk for severe infections in the first year of life. Journal of tropical pediatrics. 2012;58(6):505-8.

11. Afran L, Garcia Knight M, Nduati E, Urban B, Heyderman R, Rowland-Jones S. HIV-exposed uninfected children: a growing population with a vulnerable immune system? Clinical \& Experimental Immunology. 2014;176(1):11-22. 
12. Heidari S, Mofenson L, Cotton MF, Marlink R, Cahn P, Katabira E. Antiretroviral drugs for preventing mother-to-child transmission of HIV: a review of potential effects on HIV-exposed but uninfected children. JAIDS Journal of Acquired Immune Deficiency Syndromes. 2011;57(4):290-6.

13. Williams PL, Hazra R, Van Dyke RB, Yildirim C, Crain MJ, SEAGE III GR, et al. Antiretroviral Exposure During Pregnancy and Adverse Outcomes in HIV-exposed Uninfected Infants and Children Using a Trigger-based Design: The SMARTT Study. AIDS (London, England). 2016;30(1):133.

14. Sugandhi N, Rodrigues J, Kim M, Ahmed S, Amzel A, Tolle M, et al. HIV Exposed Infants: Rethinking care for a lifelong condition. AIDS (London, England). 2013;27(0 2):S187.

15. Udeagu C-CN, Webster TR, Bocour A, Michel P, Shepard CW. Lost or just not following up: public health effort to re-engage HIV-infected persons lost to follow-up into HIV medical care. Aids. 2013;27(14):2271-9.

16. Bigna JJR, Noubiap JJN, Kouanfack C, Plottel CS, Koulla-Shiro S. Effect of mobile phone reminders on follow-up medical care of children exposed to or infected with HIV in Cameroon (MORE CARE): a multicentre, single-blind, factorial, randomised controlled trial. The Lancet infectious diseases. 2014;14(7):600-8.

17. Lester RT, Ritvo P, Mills EJ, Kariri A, Karanja S, Chung MH, et al. Effects of a mobile phone short message service on antiretroviral treatment adherence in Kenya (WelTel Kenya1): a randomised trial. The Lancet. 2010;376(9755):1838-45.

18. Dillingham R, Ingersoll K, Flickinger TE, Waldman AL, Grabowski M, Laurence C, et al. PositiveLinks: a mobile health intervention for retention in HIV care and clinical outcomes with 12-month follow-up. AIDS patient care and STDs. 2018;32(6):241-50.

19. Tejiokem MC, Faye A, Penda IC, Guemkam G, Ndongo FA, Chewa G, et al. Feasibility of early infant diagnosis of HIV in resource-limited settings: the ANRS 12140-PEDIACAM study in Cameroon. PLoS One. 2011;6(7):e21840.

20. Sofeu CL, Warszawski J, Ndongo FA, Penda IC, Ndiang ST, Guemkam G, et al. Low birth weight in perinatally HIV-exposed uninfected infants: observations in urban settings in Cameroon. PloS one. 2014;9(4):e93554.

21. Lee H, Hogan JW, Genberg BL, Wu XK, Musick BS, Mwangi A, et al. A state transition framework for patient-level modeling of engagement and retention in HIV care using longitudinal cohort data. Statistics in medicine. 2018;37(2):302-19.

22. Sidze LK, Faye A, Tetang SN, Penda I, Guemkam G, Ateba FN, et al. Different factors associated with loss to follow-up of infants born to HIV-infected or uninfected mothers: observations from the ANRS 12140-PEDIACAM study in Cameroon. BMC public health. 2015;15(1):228.

23. Blanco AJ, Micek MA, Frenkel LM, Montoya P, Karagianis M, Matunha L, et al. Loss to follow-up among HIV-exposed children in an HIV Clinic in Beira, Mozambique. SAGE Open. 2015;5(3):2158244015590841.

24. Pop-Eleches C, Thirumurthy H, Habyarimana JP, Zivin JG, Goldstein MP, De Walque D, et al. Mobile phone technologies improve adherence to antiretroviral treatment in a resource-limited setting: a 
randomized controlled trial of text message reminders. AIDS (London, England). 2011;25(6):825.

25. Nordentoft PB, Engell-Sørensen T, Jespersen S, Correia FG, Medina C, da Silva Té D, et al. Assessing factors for loss to follow-up of HIV infected patients in Guinea-Bissau. Infection. 2017;45(2):187-97.

26. Bwirire L, Fitzgerald M, Zachariah R, Chikafa V, Massaquoi M, Moens $M$, et al. Reasons for loss to follow-up among mothers registered in a prevention-of-mother-to-child transmission program in rural Malawi. Transactions of the royal society of tropical medicine and hygiene. 2008;102(12):1195-200.

27. Geng EH, Bangsberg DR, Musinguzi N, Emenyonu N, Bwana MB, Yiannoutsos CT, et al. Understanding reasons for and outcomes of patients lost to follow-up in antiretroviral therapy programs in Africa through a sampling-based approach. Journal of acquired immune deficiency syndromes (1999). 2010;53(3):405.

28. Braitstein P, Katshcke A, Shen C, Sang E, Nyandiko W, Ochieng VO, et al. Retention of HIV-infected and HIV-exposed children in a comprehensive HIV clinical care programme in Western Kenya. Tropical Medicine \& International Health. 2010;15(7):833-41.

29. Jong S, Cuca Y, Thompson LM. Meta-analysis of mobile phone reminders on HIV patients' retention to care. Journal of mobile technology in medicine. 2017;6(1):5.

\section{Tables}


Table 1

Proportion of NC children at the start of evaluation (31 March 2014)

\begin{tabular}{|c|c|c|c|c|c|c|c|}
\hline & \multicolumn{2}{|c|}{ Compliant } & \multicolumn{2}{|c|}{$\begin{array}{l}\text { Non- } \\
\text { compliant }\end{array}$} & \multicolumn{3}{|c|}{ Total } \\
\hline & $\mathbf{N}$ & $\%$ & $\mathbf{N}$ & $\%$ & $\mathbf{N}$ & $\%$ & P-value \\
\hline & 386 & 70.7 & 160 & 29.3 & 546 & 100 & \\
\hline Sites $(n=546)$ & & & & & & & $<0.001$ \\
\hline MCC-CBF & 176 & 66.4 & 89 & 33.6 & 265 & 48.5 & \\
\hline LHD & 82 & 63.6 & 47 & 36.4 & 129 & 23.6 & \\
\hline $\mathrm{EHC}$ & 128 & 84.2 & 24 & 15.8 & 152 & 27.8 & \\
\hline Groups of children $(n=546)$ & & & & & & & $<0.001$ \\
\hline HIV-infected children & 138 & 90.2 & 15 & 9.8 & 153 & 28.0 & \\
\hline HEU-children & 121 & 60.5 & 79 & 39.5 & 200 & 36.6 & \\
\hline HUU-children & 127 & 65.8 & 66 & 34.2 & 193 & 35.3 & \\
\hline Marital status of mother $(n=468)$ & & & & & & & 0.121 \\
\hline Single/Divorced/Widowed & 86 & 76.8 & 26 & 23.2 & 112 & 23.9 & \\
\hline Married/Cohabitation & 244 & 68.5 & 112 & 31.5 & 356 & 76.1 & \\
\hline Mother's education level $(n=538)$ & & & & & & & 0.574 \\
\hline None/Primary & 66 & 75 & 22 & 25 & 88 & 16.4 & \\
\hline Secondary & 218 & 69.2 & 97 & 30.8 & 315 & 58.6 & \\
\hline Higher & 95 & 70.4 & 40 & 29.6 & 135 & 25.1 & \\
\hline Mother's occupation $(n=536)$ & & & & & & & 0.229 \\
\hline At Home/Unemployment & 157 & 74.4 & 54 & 25.6 & 211 & 39.4 & \\
\hline Training/education & 70 & 69.3 & 31 & 30.7 & 101 & 18.8 & \\
\hline Paid activity & 150 & 67 & 74 & 33 & 224 & 41.8 & \\
\hline Change of residence place $(n=446)$ & & & & & & & 0.597 \\
\hline Yes & 46 & 63 & 27 & 37 & 73 & 16.4 & \\
\hline No & 250 & 67 & 132 & 33 & 373 & 83.6 & \\
\hline Mother vital status $(n=544)$ & & & & & & & 0.264 \\
\hline Died & 10 & 90.9 & 1 & 9.1 & 11 & 2.0 & \\
\hline
\end{tabular}




\begin{tabular}{|lllllllll|}
\hline & \multicolumn{3}{c}{ Compliant } & \multicolumn{2}{c|}{$\begin{array}{l}\text { Non- } \\
\text { compliant }\end{array}$} & \multicolumn{3}{c|}{ Total } \\
\hline Alive & 373 & 70.1 & 159 & 29.9 & 532 & 97.8 & \\
\hline Not know & 1 & 100.0 & 0 & 0.0 & 1 & 0.2 & \\
\hline Father vital status $(\mathbf{n = 5 4 2 )}$ & & & & & & & 0.23 \\
\hline Died & 7 & 70.0 & 3 & 30.0 & 10 & 1.8 & \\
\hline Alive & 361 & 70 & 155 & 30 & 516 & 95.2 & \\
\hline Not know & 15 & 93.7 & 1 & 6.3 & 16 & 3.0 & \\
\hline Familly composition $(\mathbf{n = 5 4 0 )}$ & & & & & & & 0.049 \\
\hline Mother & 149 & 75.6 & 48 & 24.4 & 197 & 36.5 & \\
\hline Mother \& father & 217 & 66.8 & 108 & 33.2 & 325 & 60.2 & \\
\hline Mother \& grandmother/grandmother & 7 & 63.6 & 4 & 36.4 & 11 & 2.0 & \\
\hline Uncle/aunt/cousin/nephew/institution & 7 & 100.0 & 0 & 0.0 & 7 & 1.3 & \\
\hline
\end{tabular}

Table 2

Not returning reasons among children NC at least once and children NC who didn't come back to visit during April 2014 - April 2017

\begin{tabular}{|lll|}
\hline Reasons & $\begin{array}{l}\text { Children NC at least one } \\
\mathbf{N}=\mathbf{2 4 6}(\%)\end{array}$ & $\begin{array}{l}\text { Children NC who didn't return to visit } \\
\mathbf{N}=109(\%)\end{array}$ \\
\hline Change of residence place city & $84(30.4)$ & $60(39.5)$ \\
\hline Lack of time & $65(23.6)$ & $27(17.8)$ \\
\hline Wish to stop follow-up & $32(11.6)$ & $28(18.4)$ \\
\hline Parents and/or child trip & $27(9.8)$ & $12(7.9)$ \\
\hline School attendance & $26(9.4)$ & $10(6.6)$ \\
\hline Forgetfulness & $20(7.2)$ & $6(3.9)$ \\
\hline Illness or death of a close relative & $13(4.7)$ & $6(3.9)$ \\
\hline Lack of motivation & $4(1.4)$ & $1(0.7)$ \\
\hline Others & $5(1.4)$ & $2(1.3)$ \\
\hline Total & $276(100)$ & $152(100)$ \\
\hline
\end{tabular}


Table 3

State transition matrix over follow-up period before call reminder enhancement. Missed visit, and Non-compliant represent respectively the missing visit for short and long term.

\begin{tabular}{|lllll|}
\hline \multicolumn{5}{|l|}{ State at $\mathrm{t} j$} \\
\hline State at tj-1 & Seen & Missed visit & Non-compliant & Deceased \\
\hline Seen & 0.84 & 0.15 & 0 & 0.11 \\
\hline Missed-visit & 0.5 & 0 & 0.47 & 0.036 \\
\hline Non-Compliant & 0.13 & 0 & 0.86 & 0.008 \\
\hline Deceased & 0 & 0 & 0 & 0 \\
\hline
\end{tabular}

Table 4

State transition matrix over follow-up period during call reminder enhancement period. Missed visit, and Non-compliant represent respectively the missing visit for short and long term.

\begin{tabular}{|lllll|}
\hline \multicolumn{5}{|c|}{ State at $\mathrm{t} j$} \\
\hline State at tj-1 & Seen & Missed visit & Non-compliant & Deceased \\
\hline Seen & 0.89 & 0.11 & 0 & 0.001 \\
\hline Missed-visit & 0.46 & 0 & 0.54 & 0.006 \\
\hline Non-compliant & 0.08 & 0 & 0.92 & 0 \\
\hline Deceased & 0 & 0 & 0 & 0 \\
\hline
\end{tabular}


Table 5

Relative risk ratios for effect of covariates on transition from seen to missed visit or deceased, relative to remaining seen, ANRS-Pediacam cohort 20142017, Cameroon

\begin{tabular}{|lll|}
\hline State tj-1 & Seen & \\
\hline State tj & Missing-visit & Deceased \\
\hline Call reminder enhancement & $0.35[0.24-0.52]^{\star \star \star}$ & $0.37[0.02-5.66]$ \\
\hline Group & & \\
\hline HIV-infected & $0.65[0.53-0.82]^{\star \star \star}$ & $12.32[3.54-42.85]^{\star \star *}$ \\
\hline HEU & REF & REF \\
\hline HUU & $1.02[0.85-1.24]$ & $0.99[0.20-5.00]$ \\
\hline Year of inclusion & & \\
\hline 2008 & REF & \\
\hline 2009 & $0.83[0.66-1.07]$ & $0.38[0.15-0.93]^{\star}$ \\
\hline 2010 & $0.83[0.63-1.08]$ & $0.83[0.36-1.94]$ \\
\hline 2011 & $1.7[1.11-2.61]^{\star}$ & $0.93[0.31-2.83]$ \\
\hline Mother Education levels & & \\
\hline None/Primary & $0.89[0.70-1.12]$ & $0.95[0.44-2.03]$ \\
\hline Secondary or Higher & REF & REF \\
\hline Mother's occupation & & $1.46[0.57-3.78]$ \\
\hline At Home/Unemployment & $0.90[0.75-1.08]$ & REF \\
\hline Training/education & $1.12[0.90-1.40]$ & \\
\hline Paid activity & REF & \\
\hline ***: pvalue< 0.001; **: pvalue $<0.01 ; *: p v a l u e<0.05$ & \\
\hline & & \\
\hline
\end{tabular}


Table 6

Relative risk ratios for effect of covariates on transition from Missed visit to Non-compliant or deceased, relative to remaining seen

\begin{tabular}{|c|c|c|}
\hline State tj-1 & Missed visit & \\
\hline State tj & Non-compliant & Deceased \\
\hline Call reminder enhancement & $0.72[0.36-1.44]$ & $1.74[0.02-111]$ \\
\hline \multicolumn{3}{|l|}{ Groupe } \\
\hline HIV-infected & $0.14[0.08-0.23]^{\star \star \star}$ & $8.76[1.60-47.9]^{\star}$ \\
\hline HEU & REF & REF \\
\hline HUU & $0.65[0.44-0.94]^{\star}$ & $0.17[-]$ \\
\hline \multicolumn{3}{|l|}{ Site } \\
\hline MCC-CBF & REF & REF \\
\hline $\mathrm{EHC}$ & $1.74[1.66-2.60] \star \star$ & $2.53[0.54-11.8]$ \\
\hline LHD & $0.55[0.37-0.82]^{\star \star}$ & $3.05[0.83-11.8]$ \\
\hline \multicolumn{3}{|l|}{ Year of inclusion } \\
\hline 2008 & REF & REF \\
\hline 2009 & $0.60[0.33-0.95]^{\star}$ & $1.79[0.32-9.96]$ \\
\hline 2010 & $0.60[0.35-1.04]$ & $1.57[0.26-9.40]$ \\
\hline 2011 & $1.59[0.67-3.76]$ & $2.73[0.33-22.71]$ \\
\hline \multicolumn{3}{|l|}{ Mother Education levels } \\
\hline None/Primary & $1.14[0.69-1.89]$ & $1.06[0.31-3.56]$ \\
\hline Secondary/Higher & REF & REF \\
\hline \multicolumn{3}{|l|}{ Mother's occupation } \\
\hline At Home/Unemployment & & $3.24[1.8-13.0]^{\star}$ \\
\hline Training/education & & $0.99[0.09-11.21]$ \\
\hline Paid activity & & REF \\
\hline$\star \star \star:$ pvalue $<0.001 ; * \star$ : pvalu & $<0.01 ; *$ : pvalue $<0$ & \\
\hline
\end{tabular}


Table 7

Relative risk ratios for effect of covariates on transition from Non-compliant to non-compliant relative to remaining seen

\begin{tabular}{|ll|}
\hline State at Tj-1 & Non-Compliant \\
\hline State at tj & Non-Compliant \\
\hline Call reminder enhancement \& & $0.57[0.26-1.23]$ \\
\hline Group & \\
\hline HIV-infected & $1.05[0.52-2.14]$ \\
\hline HEU & REF \\
\hline HUU & $0.61[0.38-1.00]$ \\
\hline Family income's $(X C F A)$ & \\
\hline$<50000$ & $2.81\left[1.34-5.92{ }^{\star}\right.$ \\
\hline $50000-150000$ & $1.53[0.85-2.75]$ \\
\hline$>150000$ & REF \\
\hline$* \star *:$ pvalue $<0.001 ; * *$ pvalue $<0.01 ; *:$ pvalue $<0.05$ \\
\hline
\end{tabular}

Figures 


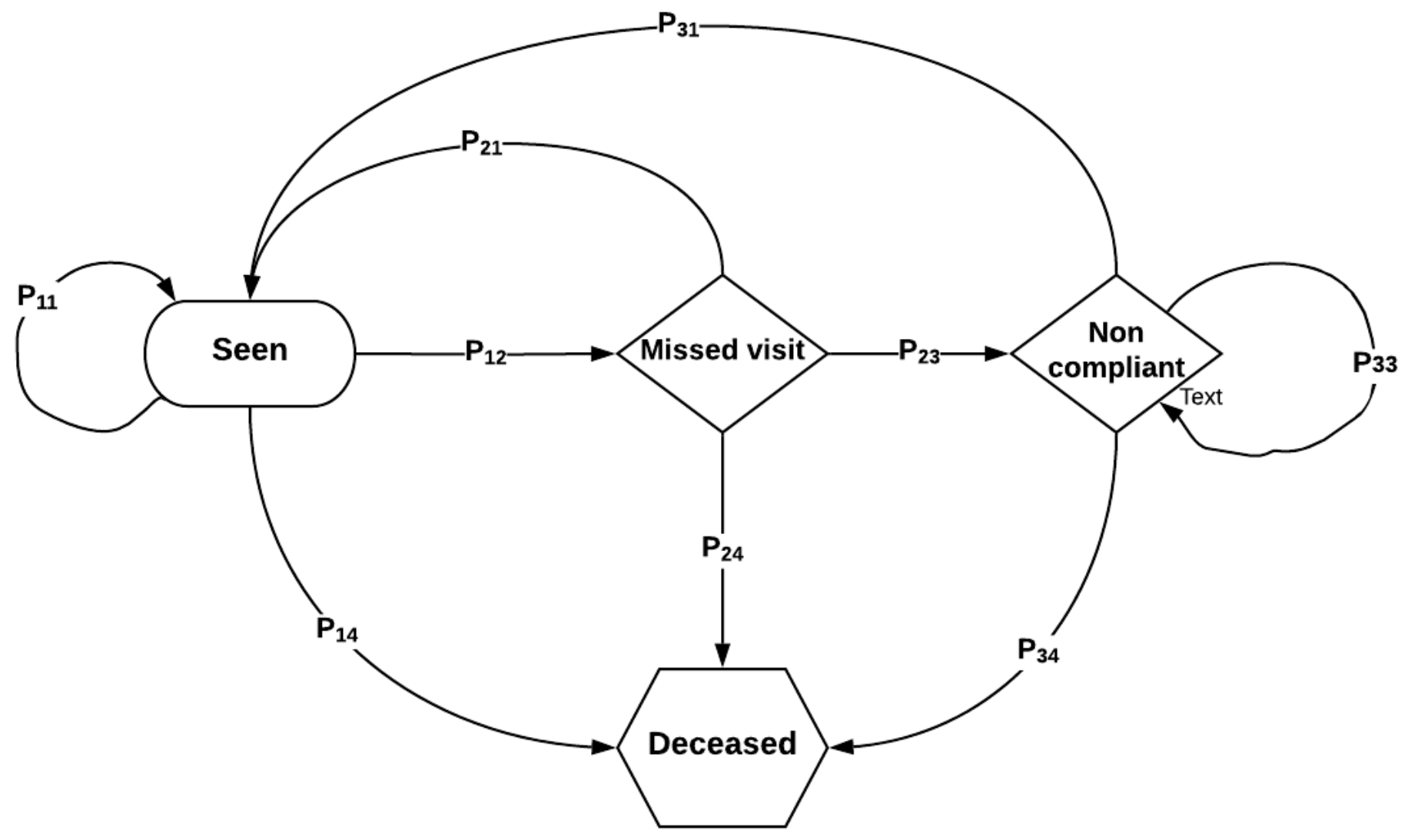

Figure 1

Cascade of state transition (Seen, missed visit, Non-compliant, deceased) during follow-up of HIVinfected children followed in Pediacam cohort. $P_{-}$ij represent the transition probability from state i to state $j$ during the follow-up period, where, $i, j=1, \ldots, 4$ are the recoded state: $1=$ Seen; $2=$ missed visit; $3=$ Noncompliant; 4=Deceased. 

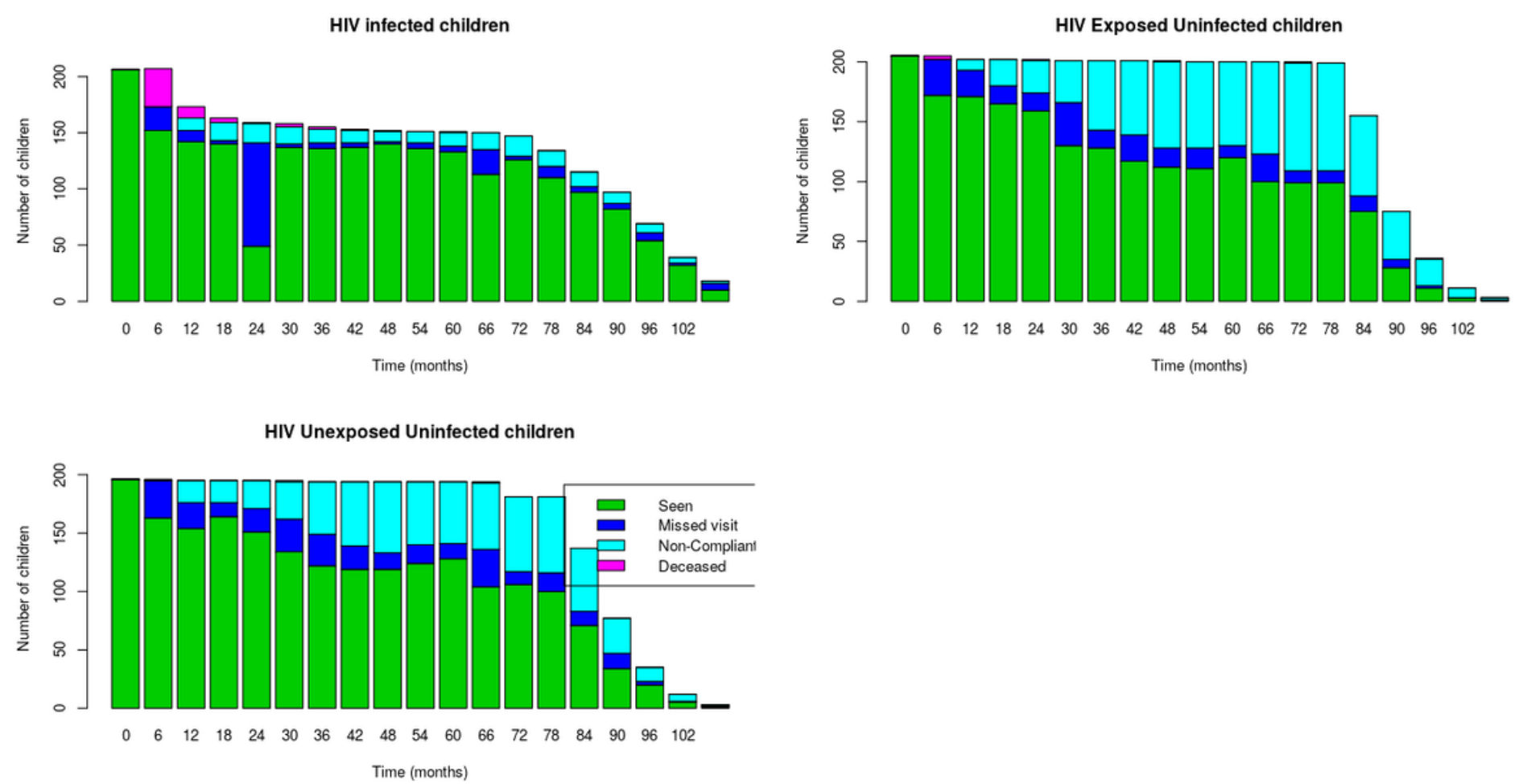

\section{Figure 2}

Number of children in each state over time.

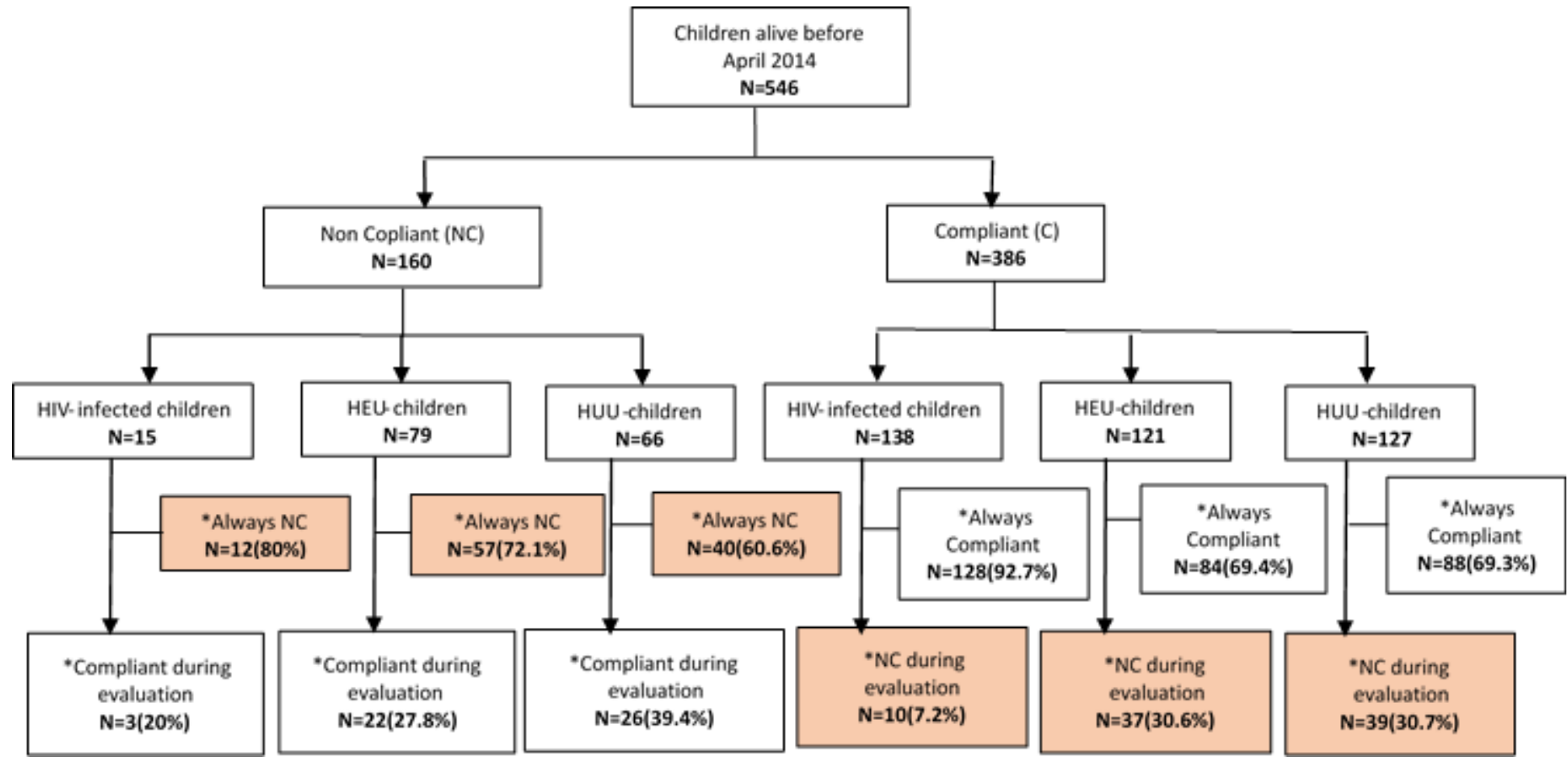

\section{Figure 3}

NC trajectory (flow) for children between April 2014 and April 2017, ANRS-Pediacam, Cameroon * Always NC: Designates among those who missed their last visit at the start, those who have never returned 
throughout the evaluation period. *Always Compliant: Designates among the Compliant at the start, those who have always kept compliant status throughout the evaluation period. ${ }^{*}$ Compliant during evaluation: Designates among those who missed their last visit at the start, those who have returned to at least one visit in the evaluation period.

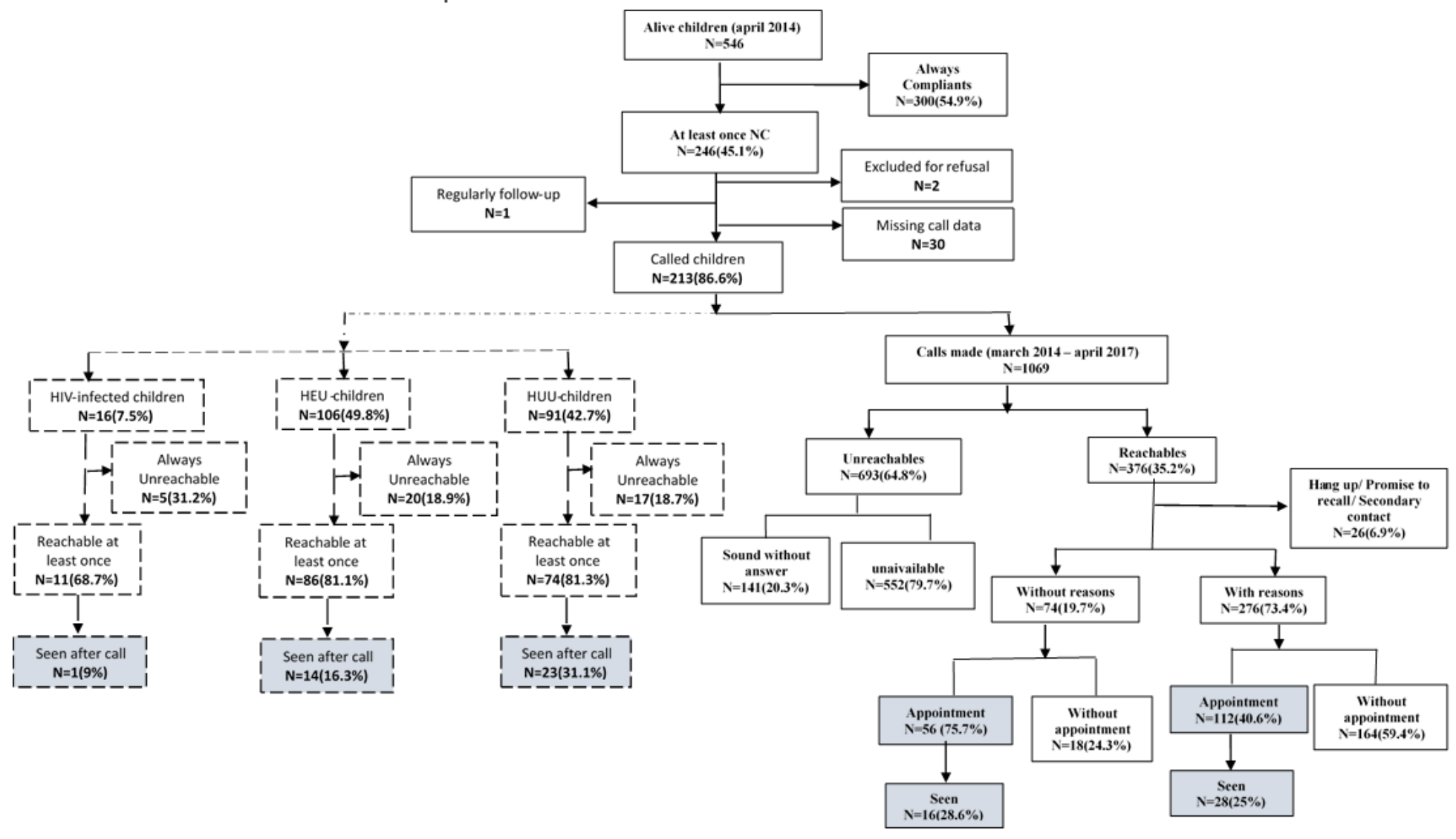

Figure 4

calls distribution by group and status 